\title{
Dynamic polarizability of rotating particles in electrorheological fluids
}

\author{
J. J. Xiao*, ${ }^{1}$ J. P. Huang, ${ }^{2}$ and K. W. $\mathrm{Yu}^{\dagger 1,3}$ \\ ${ }^{1}$ Department of Physics, The Chinese University of Hong Kong, Shatin, New Territories, Hong Kong, China \\ ${ }^{2}$ Surface Physics Laboratory (National Key Laboratory) and Department of Physics, Fudan University, Shanghai 200433, China \\ ${ }^{3}$ Institute of Theoretical Physics, The Chinese University of Hong Kong, Shatin, New Territories, Hong Kong, China
}

(Dated: April 26, 2022)

\begin{abstract}
A rotating particle in electrorheological (ER) fluid leads to a displacement of its polarization charges on the surface which relax towards the external applied field $\mathbf{E}_{0}$, resulting in a steadystate polarization at an angle with respect to $\mathbf{E}_{0}$. This dynamic effect has shown to affect the ER fluids properties dramatically. In this paper, we develop a dynamic effective medium theory (EMT) for a system containing rotating particles of finite volume fraction. This is a generalization of established EMT to account for the interactions between many rotating particles. While the theory is valid for three dimensions, the results in a special two dimensional configuration show that the system exhibits an off-diagonal polarization response, in addition to a diagonal polarization response, which resembles the classic Hall effect. The diagonal response monotonically decreases with an increasing rotational speed, whereas the off-diagonal response exhibits a maximum at a reduced rotational angular velocity $\omega_{0}$ comparing to the case of isolated rotating particles. This implies a way of measurement on the interacting relaxation time. The dependencies of the diagonal and off-diagonal responses on various factors, such as $\omega_{0}$, the volume fraction, and the dielectric contrast, are discussed.
\end{abstract}

Keywords: Electrorheological fluid; Dynamic polarizability; Effective medium theory; Off-diagonal response

\section{INTRODUCTION}

In many aspects, an electrorheological (ER) fluid containing suspensions at rest is quite different from an ER fluid containing suspensions subjected to rotational motions, $[1,2,3,4,5,6,7,8]$ which has been referred to the dynamic effect in ER fluid, $[9,10,11]$ and is of great relevance in various applications of ER fluid. Reports regarding this dynamic effect have been extensive in the literature. $[12,13,14,15,16,17,18]$ Typically there are three cases [11] for rotation of the suspending particles about their centers: (1) rotation due to external torque, which, for instance, might be induced by the shear flow in the ER fluid. [3, 9, 10, 11, 12, 13] (2) particle rotation due to a rotating external field, which is a common phenomena utilized in electrorotation assay. $[14,15](3)$ spontaneous rotation of particular particles within weak conducting fluid in a dc field above a threshold. This is first discovered almost a century ago, [19] now recognized as Quincke effect or negative ER effect. [16, 17] In all these cases, the rotation axis is favored in a special direction.

The rotational motion leads to a displacement of surface charge (not for Quincke rotation) on the particles that mediates the interactions in the ER system. For an isolated rotating particle, it has been shown that the displaced surface charge on the particle due to rotation

\footnotetext{
*Electronic mail: jjxiao@phy.cuhk.edu.hk

Present address: Department of Physics, The Hong Kong University of Science and Technology, Clear Water Bay, Kowloon, Hong Kong, China

†Electronic mail: kwyu@phy.cuhk.edu.hk
}

will relax towards the external applied field $\mathbf{E}_{0}$. [9] The result of competition between the displacement and the relaxation is a steady-state polarization which deviates from the applied field's direction, and depends on the product of the rotational angular velocity $\boldsymbol{\omega}_{0}$ of the particle and the relaxation time $\tau_{0}$. At the same time, the magnitude of the steady-state polarization is reduced by a factor as compared to the resting case. [5, 9] which is determined by the same competition. As the interparticle force depends crucially on the magnitude and direction of polarizations, the complications due to the rotational motion were shown to be dramatic. [10, 18] A first attempt of two rotating particles was achieved by Wan et al. [9] However, a more serious attempt must be directed to a suspension of finite volume fraction. It is therefore instructive to extend the existing theories to the scenario of many rotating particles, as in realistic ER suspensions. In which case, it is necessary to assess the local field effects.

However, for an individual rotating particle in such a system, e.g., see Fig. 1, the relaxation of the induced surface charge on the particle will be towards the sum $\mathbf{E}_{\text {total }}$ of the external applied field $\mathbf{E}_{0}$ and the local field $\mathbf{E}_{\mathrm{L}}$ due to all other particles. Moreover, the presumable polarization $\mathbf{P}$ of the particles is generally not parallel to the applied field $\mathbf{E}_{0}$ [Fig. 1(b)]. In a heuristic example, since the local field is always proportional to the polarization $\mathbf{P}$ by virtual of the Lorentz concept, the total field is neither along the polarization nor along the external applied field in presence of any rotational motion. The result of the competition between rotation and relaxation, however, must lead to the prescribed steady-state polarization. As a result, the steady-state polarization must be determined self-consistently and it leads to an effective medium theory for such a system of many rotating 
particles. Regarding the local field effects, the difficulty lies in the tensorial nature of the problem. More precisely, as we mentioned, it is because the polarization is not parallel to the applied field due to the competition between the displaced surface charge on the particles via mechanical rotation and the polarization's relaxation towards the applied field. Therefore, a successful theory must accommodate these properties correctly.

In the next section, we shall put forth an effective medium theory that is able to describe such properties of ER fluid consisting rotating suspensions in a very general situation. Then in Sec. III we examine in detail a special case of two-dimensional (2D) configuration and present some simple analytical results which will be confirmed numerically. At the same time, we report an interesting off-diagonal polarization response arising from the dynamic effect. We conclude the paper with some discussions in the last section.

\section{DYNAMIC EFFECTIVE MEDIUM THEORY}

Let us consider a volume fraction $\rho$ of homogeneous spherical particles of dielectric constant $\epsilon_{1}$, embedded in a host medium of unit dielectric constant [see Fig. 1(a)]. An external electric field $\mathbf{E}_{0}$ is applied to the system. We then assume a uniform rotational angular velocity $\boldsymbol{\omega}_{0}$ for all the suspending particles. This could be a typical ER fluid under shear, [8] wherein the suspended particles are of micrometeric size and have a large ratio of surface-tobulk polarization.

If we first ignore the interaction between the particles, the dynamic equation for the induced dipole moment $\mathbf{p}$ of a single particle is given by [9]

$$
\dot{\mathbf{p}}+\boldsymbol{\omega}_{0} \times \mathbf{p}=\frac{1}{\tau_{0}}\left(\mathbf{p}-\mathbf{p}^{(0)}\right),
$$

where $\mathbf{p}^{(0)}$ is the dipole moment of the particle in the absence of rotation, and $\tau_{0}$ is the relaxation time of the noninteracting particles. The steady-state dipole moment of the particle can be expressed in terms of $\mathbf{p}^{(0)}$

$$
\mathbf{p}=\frac{\mathbf{p}^{(0)}+\tau_{0}^{2} \boldsymbol{\omega}_{0}\left(\boldsymbol{\omega}_{0} \cdot \mathbf{p}^{(0)}\right)+\tau_{0}\left(\boldsymbol{\omega}_{0} \times \mathbf{p}^{(0)}\right)}{1+\left(\omega_{0} \tau_{0}\right)^{2}}
$$

where $\omega_{0}=\left|\boldsymbol{\omega}_{0}\right|$. The steady-state dipole moment $\mathbf{p}$ of Eq. (2) is generally expressed in a sum of axial and solenoidal terms. Furthermore, we can show that

$$
\boldsymbol{\omega}_{0} \cdot \mathbf{p}^{(0)}=\boldsymbol{\omega}_{0} \cdot \mathbf{p}
$$

In the presence of interaction, one can define the effective dielectric constant $\boldsymbol{\epsilon}$ in terms of the polarization $\mathbf{P}=$ $\sum_{i} \mathbf{p}_{i} / V$ of the system [20]

$$
\mathbf{P}=\frac{\boldsymbol{\epsilon}-\boldsymbol{I}}{4 \pi} \cdot \mathbf{E}_{0}
$$
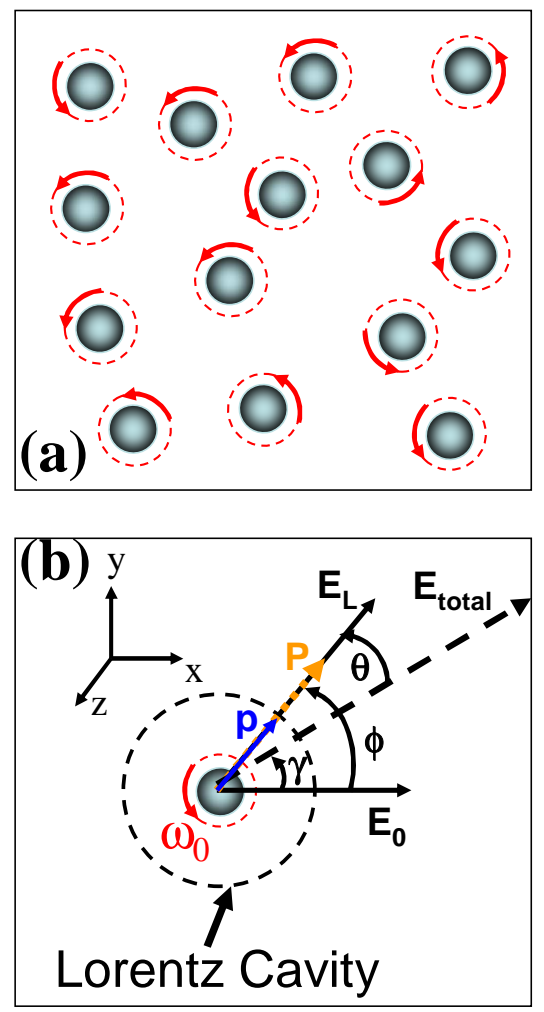

FIG. 1: (Color online) (a) Cartoon representation for a system containing rotating particles of finite volume fraction. (b) Schematic relationship of the single particle polarization and the self-consistent macroscopic polarization.

where $\boldsymbol{I}$ is the identity tensor. Note that by definition, $\boldsymbol{\epsilon}$ is a tensor and frequency dependent. By invoking the Lorentz local electric field for the steady-state responses, the induced dipole moment is given by

$$
\mathbf{p}=\mathbf{P} v=\boldsymbol{\alpha} \cdot\left(\mathbf{E}_{0}+\frac{4 \pi}{3} \mathbf{P}\right),
$$

where $\boldsymbol{\alpha}$ is polarizability tensor of the rotating particle which is also frequency dependent, and $v$ is the average volume per particle in the suspension. By substituting Eq. (4) into Eq. (5), we arrive at the dynamic MaxwellGarnett formula

$$
\boldsymbol{\epsilon}-\boldsymbol{I}=\frac{4 \pi}{3 v} \boldsymbol{\alpha} \cdot(\boldsymbol{\epsilon}+2 \boldsymbol{I}) .
$$

Thus Eq. (6) represents a generalization of the static Maxwell-Garnett approximation (MGA) to that of rotating particles. In the absence of rotation, the polarizability tensor $\boldsymbol{\alpha}^{(0)}=\alpha^{(0)} \boldsymbol{I}$ becomes diagonal

$$
\alpha^{(0)}=\frac{\epsilon_{1}-1}{\epsilon_{1}+2} a^{3},
$$

where $a$ is the radius of particle and $\epsilon_{1}$ is the dielectric constant of the particles. Thus $\boldsymbol{\epsilon}^{(0)}=\epsilon^{(0)} \boldsymbol{I}$ can be ob- 
tained from the (static) Maxwell-Garnett formula:

$$
\frac{\epsilon^{(0)}-1}{\epsilon^{(0)}+2}=\rho\left(\frac{\epsilon_{1}-1}{\epsilon_{1}+2}\right)
$$

where $\rho=4 \pi a^{3} / 3 v$ is the volume fraction. Since $\mathbf{p}$ can be expressed in terms of $\mathbf{p}^{(0)}, \boldsymbol{\alpha}$ can be expressed in terms of $\boldsymbol{\alpha}^{(0)}$

$$
\boldsymbol{\alpha}=\frac{\boldsymbol{I}+\tau_{0}^{2} \boldsymbol{\omega}_{0} \boldsymbol{\omega}_{0}+\tau_{0}\left(\boldsymbol{\omega}_{0} \times \boldsymbol{I}\right)}{1+\left(\omega_{0} \tau_{0}\right)^{2}} \cdot \boldsymbol{\alpha}^{(0)}=\boldsymbol{A} \cdot \boldsymbol{\alpha}^{(0)},
$$

where $\boldsymbol{\omega}_{0} \boldsymbol{\omega}_{0}$ represents a dyadic tensor. It is worthy noting that the above derivation and the resulting Eq. (9) are independent of coordinates and rotationally invariant in space. Specifically, the most general form of $\boldsymbol{A}$ reads, for example, in the coordinate represented by the inset of Fig. 1(b)

$$
\boldsymbol{A}=\frac{1}{1+\left(\omega_{0} \tau_{0}\right)^{2}}\left[\left(\begin{array}{ccc}
1+\omega_{x}^{2} \tau_{0}^{2} & \omega_{x} \omega_{y} \tau_{0}^{2} & \omega_{x} \omega_{z} \tau_{0}^{2} \\
\omega_{x} \omega_{y} \tau_{0}^{2} & 1+\omega_{y}^{2} \tau_{0}^{2} & \omega_{y} \omega_{z} \tau_{0}^{2} \\
\omega_{x} \omega_{z} \tau_{0}^{2} & \omega_{y} \omega_{z} \tau_{0}^{2} & 1+\omega_{z}^{2} \tau_{0}^{2}
\end{array}\right)+\left(\begin{array}{ccc}
0 & -\omega_{z} \tau_{0} & \omega_{y} \tau_{0} \\
\omega_{z} \tau_{0} & 0 & -\omega_{x} \tau_{0} \\
-\omega_{y} \tau_{0} & \omega_{x} \tau_{0} & 0
\end{array}\right)\right]
$$

where $\omega_{0}^{2}=\omega_{x}^{2}+\omega_{y}^{2}+\omega_{z}^{2}$. Thus, the self-consistent equation [Eq. (6)] becomes simultaneous equations for the components of $\boldsymbol{\epsilon}$, which are generally quite complicated.

\section{SPECIAL CONFIGURATION IN 2D}

The solenoidal term consisting of the cross product $\boldsymbol{\omega}_{0} \times \mathbf{p}^{(0)}$ in the numerator of Eq. (2) indicates a derogation of the dynamic effect in the direction parallel to $\boldsymbol{\omega}_{0}$. Thus Eq. (6) can be discussed in a $2 \mathrm{D}$ case if $\mathbf{E}_{0}$ is parallel to one of $\omega_{x}, \omega_{y}$, and $\omega_{z}$. In other words, one

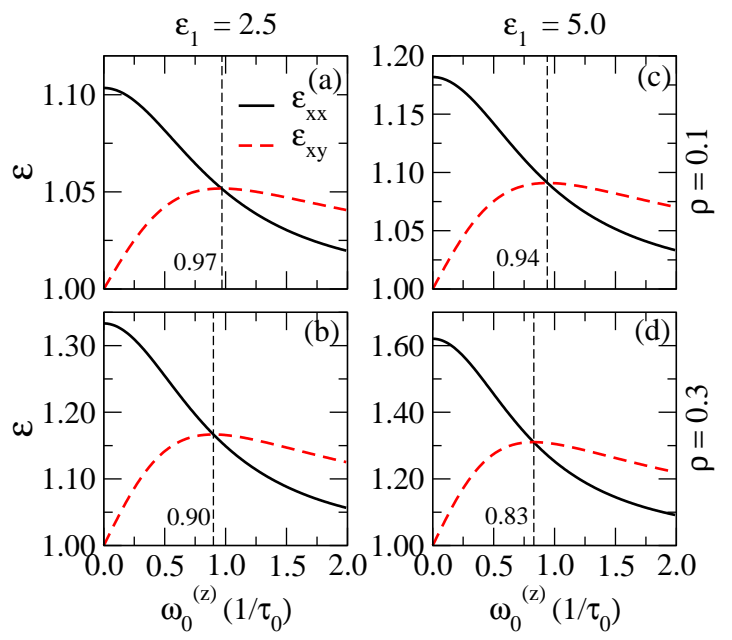

FIG. 2: (Color online) The diagonal $\left(\epsilon_{x x}\right)$ and off-diagonal $\left(\epsilon_{x y}\right)$ components of the effective dielectric constant $\epsilon$ for the case of uniform rotation of angular velocity $\boldsymbol{\omega}_{0}=\omega_{0} \hat{z}$. Note that there is a maximum off-diagonal response where $\epsilon_{x x}=$ $\epsilon_{x y}$, and the peak position $\omega_{0}^{*}$ (marked by the vertical dashed lines) of $\epsilon_{x y}$ shifts to the lower part as the volume fraction $\rho$ or the dielectric contrast $\epsilon_{1}$ increases. can always decompose $\boldsymbol{\omega}_{0}$ to two parts, parallel and perpendicular to $\mathbf{E}_{0}$. Due to this fact, it is instructive to simply consider a special geometry in which $\omega_{0}=\omega_{0} \hat{z}$, while $\mathbf{p}$ lies in the $x y$ plane, then Eq. (9) represents a transformation $\boldsymbol{\alpha}=\boldsymbol{A}\left(\omega_{0} \tau_{0}\right) \cdot \boldsymbol{\alpha}^{(0)}$, and

$$
\boldsymbol{A}\left(\omega_{0} \tau_{0}\right)=\left(\begin{array}{ccc}
\frac{1}{1+\left(\omega_{0} \tau_{0}\right)^{2}} & -\frac{\omega_{0} \tau_{0}}{1+\left(\omega_{0} \tau_{0}\right)^{2}} & 0 \\
\frac{\omega_{0} \tau_{0}}{1+\left(\omega_{0} \tau_{0}\right)^{2}} & \frac{1}{1+\left(\omega_{0} \tau_{0}\right)^{2}} & 0 \\
0 & 0 & 1
\end{array}\right)
$$

which indicates that the rotation does not affect the polarization along $z$ axis. In this case, the self-consistent equations become

$$
\begin{aligned}
\epsilon_{x x}-1 & =\frac{\rho}{a^{3}} \frac{\alpha^{(0)}}{1+\left(\omega_{0} \tau_{0}\right)^{2}}\left(\epsilon_{x x}+2-\omega_{0} \tau_{0} \epsilon_{x y}\right), \\
\epsilon_{x y} & =\frac{\rho}{a^{3}} \frac{\alpha^{(0)}}{1+\left(\omega_{0} \tau_{0}\right)^{2}}\left[\epsilon_{x y}+\omega_{0} \tau_{0}\left(\epsilon_{y y}+2\right)\right] \\
\epsilon_{z z}-1 & =\frac{\rho}{a^{3}} \alpha^{(0)}\left(\epsilon_{z z}+2\right)
\end{aligned}
$$

while $\epsilon_{y y}=\epsilon_{x x}, \epsilon_{y x}=-\epsilon_{x y}, \epsilon_{y z}=\epsilon_{z y}=\epsilon_{x z}=\epsilon_{z x}=0$. Thus, $\epsilon_{z z}$ is described by the usual MGA, as expected. Equations (12) suggest a complex notation. If we adopt complex notation $\tilde{p}=p_{x}+i p_{y}$, the transformation $\tilde{p}=$ $\tilde{A} \tilde{p}^{(0)}$ can be achieved by a complex number $\tilde{A}=1 /(1-$ $\left.i \omega_{0} \tau_{0}\right)$. If we write $\tilde{\epsilon}=\epsilon_{x x}+i \epsilon_{x y}$, the dynamic MGA [Eq. (6)] becomes a scalar but complex equation

$$
\frac{\tilde{\epsilon}-1}{\tilde{\epsilon}+2}=\rho \tilde{A}\left(\frac{\epsilon_{1}-1}{\epsilon_{1}+2}\right) .
$$

One can show that Eqs. (6) and (13) lead to exactly the same self-consistent equations [Eqs. (12a) and (12b)] for this special configuration in $2 \mathrm{D}$. The polarization can be obtained from Eq. (4)

$$
\tilde{P}=\frac{3 \beta}{4 \pi\left(1-\beta-i \omega_{0} \tau_{0}\right)}=\frac{3 \beta /(1-\beta)}{4 \pi\left(1-i \omega_{0} \tau\right)},
$$


where

$$
\beta=\rho \frac{\epsilon_{1}-1}{\epsilon_{1}+2},
$$

and

$$
\tau=\frac{\tau_{0}}{1-\beta} .
$$

As a consequence, when we vary the rotational angular velocity $\omega_{0}, P_{y} \equiv \operatorname{Im}[\tilde{P}]$ will exhibit a peak when $\omega_{0} \tau=1$. These are clearly shown by Fig. 2, where we plot the diagonal effective dielectric constant $\epsilon_{x x}$ (solid lines) and the off-diagonal effective dielectric constant $\epsilon_{x y}$ (dashed lines) as functions of $\omega_{0}$. The effective dielectric constant can be solved directly from Eq. (6), Eq. (12), or from Eqs. (4) and (14). It is very interesting to see that an electric field $\mathbf{E}_{0}=E_{0} \hat{x}$ can induce a polarization response in the perpendicular direction $\hat{y}$, i.e., nonvanishing $P_{y}$ appears due to the rotational motion $\boldsymbol{\omega}_{0}=\omega_{0} \hat{z}$ of the particles, which accumulates part of the surface charges, or rotates the dipole moment $\mathbf{p}$, off the $\hat{x}$ direction. Due to this transfer by rotational motion, the diagonal response $\epsilon_{x x}$ decreases monotonically as the strength of the transfer effect increases (e.g., rotation speeds up), whereas a peak in $\epsilon_{x y}$ shows up. This observation of offdiagonal response is quite interesting, resembling a classic Hall effect. In Fig. 2, we also illustrate both the effects of the volume fraction $\rho$ and the dielectric contrast $\epsilon_{1}$ on the polarization responses, as well as on the peak shifting of $\epsilon_{x y}$. We have specifically used $\epsilon_{1}=2.5$ in Figs. 2(a) and 2(b) (panels at the left), while $\epsilon_{1}=5.0$ in Figs. 2(c) and 2(d) (panels at the right). Also we set $\rho=0.1$ and 0.3 for the upper panels and the lower panels, respectively. Combination of the effects of the dielectric contrast $\epsilon_{1}$ and the volume fraction $\rho$ is actually captured by the definition of $\beta$ in Eq. (15). It is clearly seen in Fig. 2 that increasing $\beta=0.033,0.057,0.1$, and 0.171 leads to a shifting of the peak position (vertical dashed lines) of $\epsilon_{x y}$ at $\omega_{0}^{*}=0.97 / \tau_{0}, 0.94 / \tau_{0}, 0.90 / \tau_{0}$, and $0.83 / \tau_{0}$. These correspond to Figs. 2(a), 2(c), 2(b), and 2(d), respectively. From another perspective, it is noticed from Eq. (16) that the relaxation time will increase from noninteracting relaxation tim $\tau_{0}$ to $\tau$ due to the interactions. This is shown in Fig. 3, in which the solid line represents Eq. (16) and the triangles $(\triangle)$ are the data of $\omega_{0}^{*}$ extracted from Fig. 2. Then we can regard $\tau$ as the interacting relaxation time, and the maximum off-diagonal response occurs when $\omega_{0} \tau=1$, which is equivalent to $\omega_{0}^{*} \tau_{0}=1$. These results suggest a way to measure the interacting relaxation time in the system.

To this end, we have demonstrated the off-diagonal response due to the rotational motion. We would also like to look upon the geometrical meaning of these results. It is easy to tell from Eq. (14) that $\tan \phi=\omega_{0} \tau$, where $\phi$ is the angle spanned by $\mathbf{E}_{0}$ and $\mathbf{E}_{\mathrm{L}}$, as shown in Fig. 1(b). Also by some simple calculations of the angles $(\phi, \theta$, and $\gamma)$ shown in Fig. 1(b) we find that $\tan \theta=\tan (\phi-\gamma)=\tan \theta_{0} \equiv \omega_{0} \tau_{0}$, where $\theta_{0}$ is the

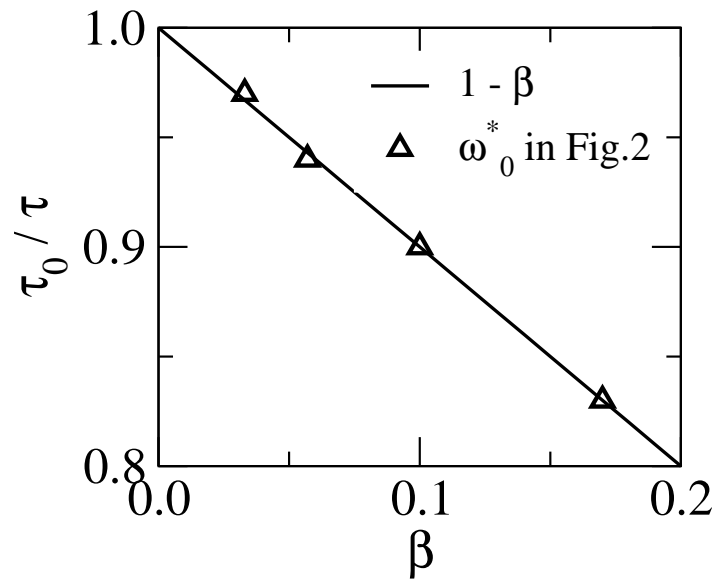

FIG. 3: Interacting relaxation time $\tau$ predicated by Eq. (16) compares favorably to numerical data extracted from Fig. 2.

angle of induced dipole moment $\mathbf{p}$ with respect to $\mathbf{E}_{0}$ for the case of an isolated rotating particle at angular velocity $\omega_{0} .[9,11]$ This is as expected and required by the self-consistency of the theory, showing that this relationship still holds for an individual rotating particle in the many-particle system: in Fig. 1(b) the dipole moment $\mathbf{p}$ and the total field $\mathbf{E}_{\text {total }}$ on this particle spans the angle $\theta=\theta_{0}$. Now it is clear that the maximum off-diagonal response appears when $\phi=\pi / 4$, where $\omega_{0}=\omega_{0}^{*}$ indicating a resonance. Right at this point, the parallel and perpendicular effective dielectric constant are equal, i.e., $\epsilon_{x x}=\epsilon_{x y}$, which is already demonstrated by Fig. 2. In the limit of $\omega_{0} \rightarrow 0, \epsilon_{x x}$ is readily predicted by the static MGA [i.e., Eq. (8)] and $\epsilon_{x y}$ vanishes. This represents the case without rotation, corresponding to $\phi \rightarrow 0$ and $\theta \rightarrow 0$ in Fig. 1(b). Whereas in the opposite limit of $\omega_{0} \rightarrow \infty$, both $\epsilon_{x x}$ and $\epsilon_{x y}$ approach unity (see also Fig. 2). This means that the rotating particle lost its tensorial polarizability $\boldsymbol{\alpha}$ of Eq. (9) [or seen from Eq. (11)] and the system dielectrically "sees" no polarizable suspension, i.e., the suspensions become "invisible", because the very fast rotational motion distributes the surface charges homogeneously along the circumference of the particle, accumulating no net charges at any interfacial place. In this case, both $\phi$ and $\theta$ lost definition because of $\mathbf{E}_{\mathrm{L}}=0$. As a result, the system responses just as the pure host media. It should be remarked that at a finite yet very fast rotation $\omega_{0} \gg 1 / \tau_{0}, \phi \rightarrow \pi / 2$ and $\theta \rightarrow 0$.

Furthermore, we examine the maximum (e.g., denoted by $\operatorname{Max}[\cdots]$ ) polarization responses of the system within the dynamic effective medium theory, in terms of both diagonal $\epsilon_{x x}$ and off-diagonal $\epsilon_{x y}$. It is quite straightforward from the previous discussion and the data shown in Fig. 2 that $\operatorname{Max}\left[\epsilon_{x x}\right]$ and $\operatorname{Max}\left[\epsilon_{x y}\right]$ occur at $\omega_{0}=0$ and $\omega_{0}=\omega_{0}^{*}$ respectively, for a certain $\beta$. This is also explicitly indicated by Eq. (14). Open circles $(\bigcirc)$ and squares $(\square)$ in Fig. 4 show the $\operatorname{Max}\left[\epsilon_{x x}\right]$ and $\operatorname{Max}\left[\epsilon_{x x}\right]$ extracted from a series figures like Fig. 2 for various $\beta$, while solid lines represent $\epsilon_{x x}\left(\omega_{0}=0\right)$ and $\epsilon_{x y}\left(\omega=\omega_{0}^{*}\right)$. The 

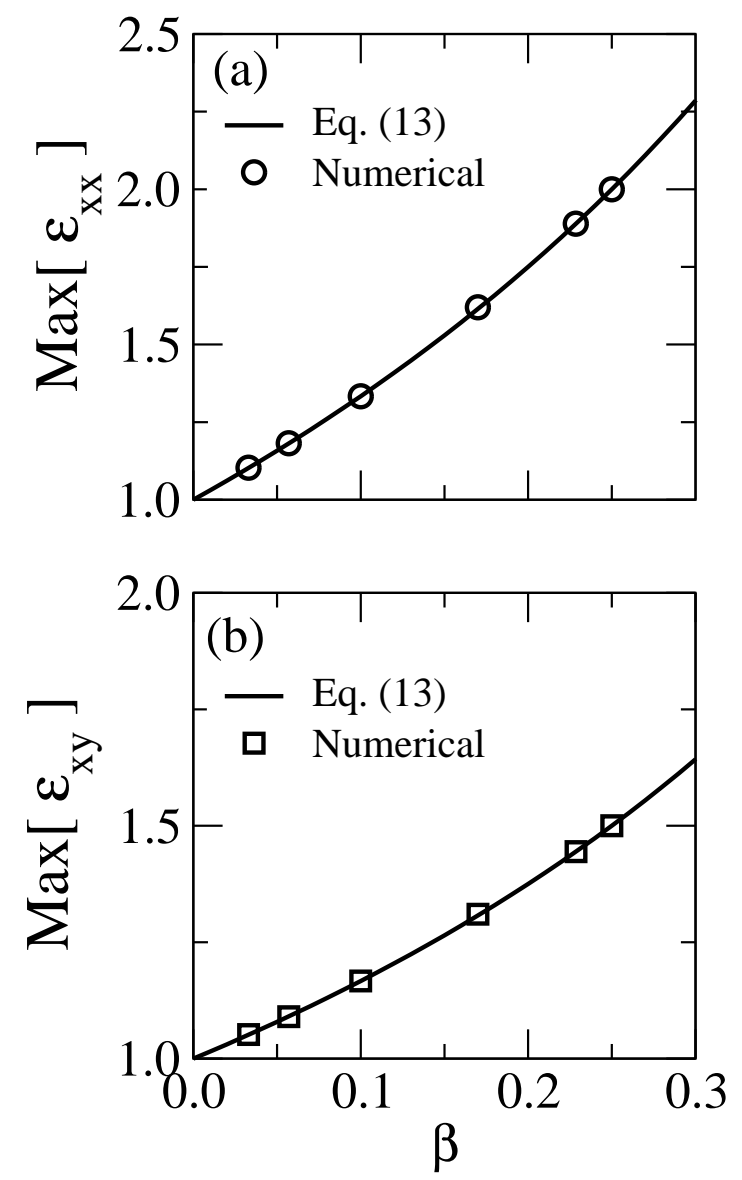

FIG. 4: The dependence of the maximum polarization responses on $\beta$. (a) $\epsilon_{x x}$ which occurs when $\omega_{0}=0$, i.e., predicted by the static MGA, and (b) $\epsilon_{x y}$ which occurs when $\omega_{0}=\omega_{0}^{*}$.

good agreements further show the usefulness of Eqs. (13) and (14) in the 2D case, which appears simple but contains enough information in understanding the interesting properties of the system. It is reasonable that both $\operatorname{Max}\left[\epsilon_{x x}\right]$ and $\operatorname{Max}\left[\epsilon_{x y}\right]$ increase monotonically with $\beta$, because commonly either an increased dielectric contrast or an increased volume fraction lead to larger effective dielectric constant.

\section{DISCUSSION AND CONCLUSION}

To conclude, we have developed an dynamic MGA to take into account the many-body interactions between rotating particles in a dynamic ER fluid. The theory, like static MGA, stems from the application of the Lorentz local field concept to a system of polarized particles in an external electric field. The only difference is now that the particles are all rotating with some angular velocity which leads to an off-diagonal polarization. We have assumed uniform rotational angular velocity in the present paper. This assumption may break down in shear flow under non-steady conditions. If the angular velocity with the same magnitude $\omega_{0}$ distributes uniformly in the solid angle, there is a reduction factor associated with the average longitudinal (diagonal) response (parallel to $\mathbf{E}_{\mathbf{0}}$ ) while the transverse (off-diagonal) responses vanish identically. Namely, $\left\langle\mathbf{p}_{\|}\right\rangle=\mathbf{p}^{(0)}\left[1-2 \omega_{0}^{2} \tau_{0}^{2} / 3\left(1+\omega_{0}^{2} \tau_{0}^{2}\right)\right]$ while $\left\langle\mathbf{p}_{\perp}\right\rangle=0$, here $\langle\cdots\rangle$ represents average over the solid angle.

Along with the present results, it is easy to derive a dynamic Bruggemann effective medium approximation (EMA). In contrast to the present asymmetric dynamic MGA, it is symmetrical and appropriate for two types of rotating particles (e.g., of $\epsilon_{1}$ and $\epsilon_{2}$ ) whose volume fractions satisfy $\rho_{1}+\rho_{2}=1$. In the same sprit of Eq. (13) in the special 2D geometry, the self-consistent equation of dynamic EMA reads

$$
\tilde{A}_{1} \rho_{1} \frac{\left(\epsilon_{1} / \tilde{\epsilon}_{e}-1\right)}{\left(\epsilon_{1} / \tilde{\epsilon}_{e}+2\right)}+\tilde{A}_{2} \rho_{2} \frac{\left(\epsilon_{2} / \tilde{\epsilon}_{e}-1\right)}{\left(\epsilon_{2} / \tilde{\epsilon}_{e}+2\right)}=0,
$$

where the two complex numbers $\tilde{A}_{1}$ and $\tilde{A}_{2}$ correspond to the transformation coefficients of two types of rotating particles. These complex numbers are assumed to be independent of the effective medium of $\boldsymbol{\epsilon}_{e}$. Physically Eq. (17) means that the induced dipole moment of type $\alpha(=1,2)$ rotating particle in an effective medium points to a different direction due to the relaxation of surface charges. However, since the different types of particles are embedded in an effective medium, the average dipole moment must vanish, thus yielding the dynamic EMA which would be applicable to analogous problems like magnetotransport in granular materials. These dynamic effective medium theories [e.g., Eq. (6) or Eq. (17)] provide guidelines for numerical simulation on the dynamic effect in ER fluids [5] and should be distinguished from those effective medium theories developed for composites of anisotropic inclusions, [21] bi-anisotropic media, [22] and chiral media, [23, 24] etc.

Extension to nonlinear ac responses $[25,26]$ is possible. In which case, peak response can be found in parallel response of higher harmonics. If we further apply an ac field at $\omega$ along the $x$ axis, then there are two characteristic responses at the sum and difference of frequencies: $\omega_{0}+\omega$, and $\omega_{0}-\omega$. When we vary $\omega$, it can be shown that $P_{y}$ exhibits either peak at $\omega_{0}+\omega_{1}$ or $\omega_{2}-\omega_{0}$. Thus, we can also determine the interacting relaxation time $\tau$ by measuring the ac responses from the relations $\left(\omega_{0}+\omega_{1}\right) \tau=1$ or $\left(\omega_{2}-\omega_{0}\right) \tau=1$. It is quite interesting that the off-diagonal polarization shows a peak below $\omega_{0}$ in the linear case while it peaks exactly at $\omega_{0}=1 / \tau_{0}$ for isolated rotating particle. So the ac field of $\omega$ can be applied perpendicular or parallel to $\mathbf{E}_{0}$. The parallel component of $\mathbf{P}$ shows no peak (it simply decrease as $\omega_{0}$ increases) in the linear case but its third harmonic term as a function of $\omega_{0}$ has a peak. [25]

Finally, we would like to discuss several complications that may introduce corrections to the local field and therefore the macroscopic responses (e.g., the effective dielectric constant $\boldsymbol{\epsilon}$ ). (1) since most ER fluid might 
be anisotropic, e.g., somehow chains are formed in the direction of applied electric field $\mathbf{E}_{0}$, the depolarization term may not be isotropic. When the lattice symmetry is lowered by an external means - under the influence of an external force/torque - the lattice is deformed, either lengthened in one direction and/or contracted in the other direction. [27] These geometric anisotropy effect will introduce a non-isotropic local field correction. [12] This additional anisotropy must also be accommodate in a consistent way. (2) The assumption of isotropic depolarization is necessary because of rotational invariance. More precisely, the Lorentz cavity is always spherical [see Fig. 1(b)]. When $\mathbf{P}$ is rotated away from $\mathbf{E}_{0}$ due to the competition between the displacement and relaxation of surface charges, the Lorentz relation $\mathbf{E}_{\mathrm{L}}=4 \pi \mathbf{P} / 3$ remains unchanged. However, the detailed dynamical correlation between the rotating particles may be further explored in presence of a distribution of rotational speed or intrinsic relaxation time. (3) There may exist certain uniaxial gradient in one direction in the system, [28] for instance, more particles aggregate in a specific part of the system. (4) We have not taken into account the effect of Brownian motion. In the presence of an external electric field, the Brownian motion would become weaker than the case of no external electric field, which is caused to appear by the electric polarization of the particle. However, competition between rotational Brownian motion and particle fibrillation is also a concern if the particles are small enough to ensure rotational diffusion.

\section{Acknowledgments}

This work was supported by the Research Grants Council Earmarked Grant of the Hong Kong SAR Government. J.P.H. acknowledges the financial support by the Pujiang Talent Project (No. 06PJ14006) of the Shanghai Science and Technology Committee, by the Shanghai Education Committee and the Shanghai Education Development Foundation ("Shu Guang" project), by Chinese National Key Basic Research Special Fund under Grant No. 2006CB921706, and by the National Natural Science Foundation of China under Grant No. 10604014 .
[1] Block, H.; Kluk, E.; McConnell, J.; Scaife, B. K. J. Colloid Interface Sci. 1984, 101, 320.

[2] Hemp, J. Proc. R. Soc. London, Ser. A 1991, 434, 297.

[3] Ladd, A. J. C. J. Chem. Phys. 1998, 88, 5051.

[4] Wahed, A. K. El.; Sproston, J. L.; Williams, E. W. J. Phys. D: Appl. Phys. 2000, 33, 2995.

[5] Dassanayake, U. M.; Offner, S. S. R.; Hu, Y. Phys. Rev. E, 2004, 69, 021507.

[6] Klingenberg, D. J.; Swol, F.; Zukoski, C. F. J. Chem. Phys. 1991, 94, 6160.

[7] Negita, K.; Ohsawa, Y. Phys. Rev. E 1995, 52, 1934.

[8] Misono, Y.; Negita, K. Phys. Rev. E 2004, 70, 061412.

[9] Wan, J. T. K.; Yu, K. W.; Gu, G. Q. Phys. Rev. E 2000, 62, 6846.

[10] Wan, J. T. K.; Yu, K. W.; Gu, G. Q. Phys. Rev. E 2001, 64, 061501.

[11] Yu, K. W.; Gu, G. Q.; Huang, J. P.; Xiao, J. J. Int. J. Mod. Phys. B 2005, 19, 1163.

[12] Cao, J. G.; Huang, J. P.; Zhou, L. W. J. Phys. Chem. B 2006, 110, 11635.

[13] Misono, Y.; Negita, K. Phys. Rev. E 2004, 70, 061412.

[14] Gimsa, J.; Muller, T.; Schnellea, T.; Fuhr, G. Biophys. J. 1996, 71, 495.

[15] Becker, F. F.; Wang, X. B.; Huang, Y.; Pethig, R.; Vyk- oukal, J.; Gascoyne, R. R. C. Proc. Natl. Acad. Sci. U.S.A. 1995, 92, 860.

[16] Cēbers, A.; Lemaire, E.; Lobry, L. Phys. Rev. E 2000, 63, 016301.

[17] Cēbers, A. Phys. Rev. Lett. 2004, 92, 034501.

[18] Tao, R.; Lan, Y. C. Phys. Rev. E 2005, 72, 041508.

[19] Quincke, G. Ann. Phys. Chem. 1896, 59, 477.

[20] Jackson, J. D. Classical Electrodynamics; Wiley: New York, 1975.

[21] Levy, O.; Stroud, D. Phys. Rev. B 1997, 568035.

[22] Weiglhofer, W. S.; Lakhtakia, A.; Michel, B. Micro. Opt. Tech. Lett. 1997, 15, 253.

[23] Sihvola, A. H.; Lindell, I. V. Elect. Lett. 1990, 26, 118.

[24] Lakhtakia, A.; Varadan, V. K.; Varadan, V. V. J. Mater. Res. 1993, 8, 917.

[25] Tian, W. J.; Huang, J. P.; Yu, K. W. Phys. Rev. E 2006, 73, 031408.

[26] Tian, W. J.; Huang, J. P.; Yu, K. W. Chem. Phys. Lett. 2006 427, 101.

[27] Lo, C. K.; Wan, J. T. K.; Yu, K. W. J. Phys.: Condens. Matter 2001, 13, 1315.

[28] Xiao, J. J.; Yu, K. W. Appl. Phys. Lett. 2006, 88, 071911. 